6

\title{
Design Innovation Guided by Modeling Assumptions
}

\author{
Jyotibikas Bhattacharya Louis Steinberg \\ Department of Computer Science \\ Rutgers University \\ New Brunswick, NJ 08903 \\ email: jyoti@paul.rutgers.edu, lou@cs.rutgers.edu
}

\begin{abstract}
In this paper we propose a method for innovation in design based on relaxing modeling assumptions. A design process is innovative if it can expand the design space it searches beyond the space defined by the initial problem formulation. Typically, an engineering design problem is formalized as a set of constraints that must be met on various properties of the resulting design (e.g., weight), and an objective function on these properties to be optimized. The properties are defined by giving a set of equations for deriving them, and the parameters in these equations define the search space. However, these equations normally are simplified models of the general underlying physics, based on various assumptions (e.g., radial symmetry) about the artifact being modeled. We present a method of expanding the initial design space, when it does not contain any acceptable designs, by introducing new parameters, based on a process of relaxing the assumptions underlying the given models of the physical properties. Our technique rationalizes the Dimensional Variable Expansion approach to innovative design found in the literature, and generalizes it.
\end{abstract}

\section{Keywords}

Design, innovation, modeling assumptions, search space, optimization 


\section{INTRODUCTION}

In order to solve an engineering design problem, it is often useful to formalize it, for instance to express it as a constrained optimization problem. Sometimes, however, it turns out that there is no acceptable solution to the problem as formalized. When this happens, a good human engineer can often find a modified formalization that opens up a larger space of designs, a space that is likely to include better solutions than the original space did, but is still constrained enough that it is feasible to find these better solutions.

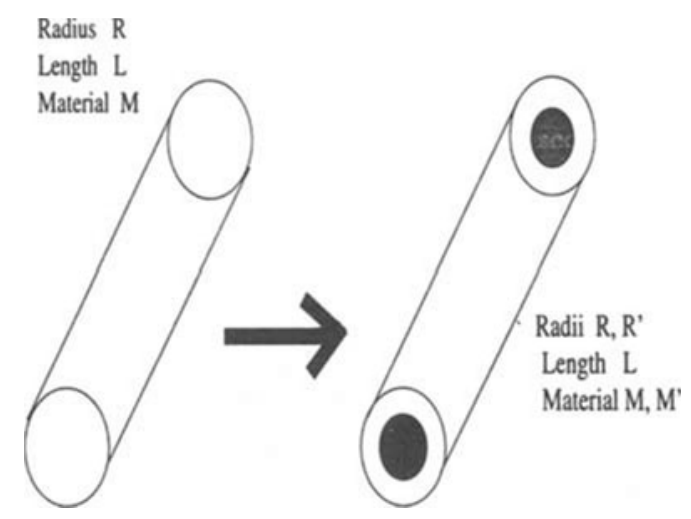

Figure 1 Innovation in Beam Design

One common way to expand a design space is to move to a richer representation of the artifact being designed. For example, if the original formalization involved a uniform cylindrical beam, representable by radius, length, and material, we might expand the design space by allowing the beam to be a composite of two materials, as in Figure 1, thus adding two new parameters to our representation: the second material and the radius at which the material changes. When a design process extends our artifact representation this way, it is referred to as innovative design ([MZG89, CA91]).

Can this kind of innovative design be automated? Yes - at least to the extent of proposing one or more larger spaces which the designer must then investigate for improved designs. A number of domain-specific heuristics have been used [ACP91, MA87], but the focus of this paper is on a domain-independent method developed by Cagan et al, called 
Dimensional Variable Expansion* (DVE) [Cag91]. In this paper, we will present an analysis that rationalizes DVE as a process of relaxing modeling assumptions, and we will show how this process can be extended to generate innovations beyond those that DVE can generate.

In the next section we will summarize the DVE method. Following sections will describe our approach and show how it accounts for the innovations DVE can handle, and will illustrate how our approach goes beyond DVE.

\section{DVE}

DVE begins with a design task formalized as a constrained optimization problem:

$$
\begin{array}{ll}
\text { Minimize: } & f(\mathbf{x}) \\
\text { Subject to: } & \mathbf{h}(\mathbf{x})=\mathbf{0} \\
\mathbf{g}(\mathbf{x}) \leq \mathbf{0}
\end{array}
$$

where $\mathbf{x}$ is a vector of variables, and $\mathbf{h}(\mathbf{x})$ and $\mathbf{g}(\mathbf{x})$ are vectors of functions.

For instance, the beam design problem mentioned above might be formalized as in Figure 2.

So far, this is a standard way of formalizing such engineering design problems. In addition, for DVE the variables must be tagged as belonging to one of several types, including the following:

- A System variable represents a quantity that depends on the "characteristic size" [ACP92] of the artifact, such as weight. ${ }^{\dagger}$

- A Region variable represents a property of a region of the artifact, e.g. $M$ and $M^{\prime}$ in the example in Figure 1, which is "not represented as an integral quantity." [Cag91]

- An Assignment variable represents a quantity that has contributions from a number of design regions. [ACP92] cites total mass as an example, but does not discuss why weight is a system variable but total mass is an assignment variable.

- a Dimensional variable is a special kind of system variable that represents the physical dimensions of a device, e.g. the length and radius in the example above.

\footnotetext{
*We will use this term to include the closely-related technique of Input Variable Expansion [ACP92]. $\dagger[$ Cag91] defines a system variable as one "defined ... as an integral quantity" over a region of the artifact, but apparently this statement is meant as a guide to the human preparing the tags, and the integral form is not given to their implementation of DVE.
} 
Minimize W, subject to:

$$
\begin{gathered}
\tau \leq \tau_{y} \\
W \leq W_{0}
\end{gathered}
$$

where

$$
\begin{gathered}
\tau=\frac{G \varphi R}{L} \\
\varphi=\frac{2 T L}{\pi G R^{4}} \\
W=\pi \rho L R^{2} \\
\rho=K_{1}(G) \\
\tau_{y}=K_{2}(G)
\end{gathered}
$$

and

$\tau$ is the shear stress at radius $R$

$\tau_{y}$ is the yield shear stress

$\varphi$ is the angle of twist

$W$ is the weight of the beam

$T$ is the external torque

$G$ is the shear modulus

$\rho$ is the the density of the material

$L$ is the length of the beam

$R$ is the radius of the beam

$K_{1}$ and $K_{2}$ represent the relations between $G$ and, respectively, $\rho$ and $\tau_{y}$, imposed by the fact that these three parameters are really all functions of the material. All that is known about $K_{1}$ and $K_{2}$ is that they are monotonicly increasing.

Figure 2 Formalization of Torsional Beam Problem

Constraints are also tagged. Serial constraints are global (e.g. a constraint on total weight), and problem-specific update formulas for each must be given to DVE to tell it how to handle them. Parallel constraints are local to a region.

Given the optimization problem and the tags, the DVE method first uses monotonicity analysis [CA91, PW79] to determine which constraints are active, i.e. are preventing further optimization of the objective function. Monotonicity analysis cannot, in general, completely determine which constraints will be active and which will not, but by imposing 
some consistency conditions it can prune out many of the possible ways of partitioning the constraints into an active set and an inactive set. DVE then considers each of the remaining, consistent, sets of active constraints as a separate case, and handles the cases in turn.

For a given case, variables which are involved both in active constraints and in the objective function are found and labeled critical. This leads to the key innovation step: if a critical variable is also a dimensional variable, the artifact is divided into regions along that dimension. E.g., in the beam problem above, if radius were identified as a critical variable, DVE will propose dividing the beam into regions such that points with $0 \leq r<R_{1}$ are in one region, points with $R_{1} \leq r<R_{2}$ are in another, etc.

When a region is split, region variables like material are duplicated, with a separate one for each region, thus leading to the new design above. DVE uses a number of transformations keyed off the variable and constraint tags to produce the complete new design probiem, including the new optimization function and constraints. In our example, this process introduces the region variable $M^{\prime}$, the material in the inner region, along with $R^{\prime}$, the radius of the inner region, as design variables, and thereby expands the space of designs to consider.

If the same constraints are active in the new problem, DVE will further divide the artifact along this dimension, and eventually will inductively decide that the constraint will always be active, and jump to the limiting case where the region property (material in our example) varies continuously along the critical dimension (here, the radius).

DVE is a powerful and general method. It has been shown capable of reinventing the I-beam and other innovations in a number of domains, including mechanical and chemical engineering. Given the right problem formulation it is even capable of inventing the wheel[CA91].

However, the phrase "given the right problem formulation" points to the main problem of DVE. As [Cag91] notes (p. 81), “... domain knowledge is required to formulate the problem for use by the technique." [emphasis added]. Thus, for instance, in the same article on p. 82-83, the weight of a beam is represented by two variables, $W^{a s s i g n}$ and $W_{1}^{\text {sys }}$. The former is the total weight over all regions, the later the weight of each region. However, in the initial design, there is only one region, and thus $W^{a s s i g n}=W_{1}^{\text {sys }}$. It might appear that the reason for including $W_{1}^{\text {sys }}$ in the formulation is the expectation that after DVE has operated there will be multiple regions, and each will need a weight variable, so the single region in the initial state must have a weight variable distinct from the global total weight.

How can the user know what tags to put on the variables without knowing in advance the innovation the system is supposed to come up with? E.g., why might we think of total 
weight as a composite property, derived by adding the weights of a number of regions of the beam? Perhaps it is because we know in general that the weight of an object is the sum of the weights of its parts, or, equivalently (taking infinitesimal "parts"), the integral of its density over its volume. True, this knowledge in not present explicitly in the design problem as formalized, but in fact it is present implicitly. Equation (5) in Figure 2 is a very specialized equation for calculating weight, applicable only to cylinders of constant density; it can be seen as a consequence of the general knowledge that weight is the integral of density, and of the specific assumptions we are making about the shape of the beam.

This insight leads us to view DVE as a special case of a more general process. The next section will describe this process and show how the innovation DVE does can be seen as a special case, and the following section will show how the approach extends DVE.

\section{INNOVATION BY RELAXING MODELING ASSUMPTIONS}

Our general process can be described, to a first approximation, as "use knowledge about modeling assumptions to guide the expansion of the design space." In order to explain this, we first need to discuss modeling assumptions and how they are related to innovation. Then we will show how they can be used to guide the expansion of the design space. After that, we will give a more detailed description of our process by showing how it can duplicate DVE's results on the torsional beam problem.

\subsection{Modeling Assumptions}

Generally, an engineering problem like the torsional beam problem above is not expressed in terms of abstract and uninterpreted variables. Rather, the constraints and the objective function are given in terms of physical properties about which an engineer has considerable knowledge, properties like weight and shear stress.

Furthermore, the equations that relate these properties are specialized forms of more general physical relationships. E.g., Equation (5) in Figure 2 gives weight as a function of the beam's radius, length, and density:

$W=\pi \rho L R^{2}$

which is a special case of the more general formula for weight:

$W=\iiint \rho(x, \alpha, r) d x d \alpha d r$ 
and Equation (4) which gives the amount the end of the beam will twist is a special case of the more general formula:

$\varphi=\frac{T L}{\int r^{2} G d A}$

The specialized equations can be derived from the more general equations given a set of assumptions about the beam:

- (A1) The beam is a cylinder with uniform radius $R$.

- (A2) The material is uniform, and hence $\tau_{y}$ and $\rho$ are independent of position ${ }^{\ddagger}$

Constraints can also be specialized. As an example, we will look in more detail at how the constraint on $\tau$ in our problem is derived. In general, the constraint would be

$\forall \alpha, r, x \quad \tau(\alpha, r, x) \leq \tau_{y}(\alpha, r, x)$

I.e., the actual shear stress at each point must not exceed yield shear stress at that point. (Yield shear stress is the stress that will break the material.) Assumption A2 above allows $\tau_{y}(\alpha, r, x)$ to become just $\tau_{y}$.

Given Assumption A1 and a general model for $\tau$, we can infer that $\tau$ is independent of $\alpha$ and $x$, and depends only on $r$, as follows:

$\tau(r)=\frac{G \varphi r}{L}$

Furthermore, from this we can see that $\tau(r)$ is largest at maximum $r$, i.e. for $r=R$, so we can use the $\tau=\tau(R)$ as an safe "overall" $\tau$. As long as this value does not exceed $\tau_{y}$, $\tau$ will not exceed $\tau_{y}$ anywhere. This gives us Equations 1 and 3 in Figure 2.

Notice that without Assumption A2, the simplified constraint on $\tau$ would be an over conservative one. It is in general possible for $\tau(R) \leq \tau_{y}(R)$ without requiring $\forall_{\tau} \tau(R) \leq \tau_{y}(r)$, but since $\tau_{y}$ is constant this cannot happen. It is the uniformity assumption that constrains the design more than is strictly necessary, by insisting that the material everywhere be strong enough to withstand the shear stress at the outer boundary.

We refer to assumptions like A1 and A2, used to derive a specialized or simplified model of a physical phenomenon, as modeling assumptions.

When DVE expands a problem's design space by introducing additional design parameters, it also must generalize the problem's analysis models, that is, the equations modeling

$\ddagger_{\text {I.E., }} \tau_{y}$ and $\rho$ are not functions of the coordinate variables $\alpha, r$, and $x$ 
physical phenomena like weight, because the modeling assumptions inherent in the initial models are violated by some designs in the expanded space. For example, the density of the beam is no longer necessarily uniform. In other words, the design space in the original problem is restricted not only by the set of parameters used to represent the design, but also by the models of physics implicit in the equations. If we expand the parameter set, we may also have to "unspecialize" the models, i.e. return them at least part way to the general models they were derived from.

\subsection{Using Modeling Assumptions to Guide Innovation}

Given the discussion above, we can explain our key idea: rather than looking at the process of relaxing modeling assumptions and revising the models as a consequence of expanding the design space, suppose we look at it as a source of guidance for expanding the design space. That is, we will look at design innovation as a process of relaxing modeling assumptions.

For instance, relaxing Assumption A2 above into

- (A2') The material and hence $\tau_{y}$ and $\rho$ are uniform across $\alpha$, and $x$, but change at some $r$.

amounts to breaking the artifact into two subregions and allowing each subregion to have differing properties, as DVE does.

A given assumption, however, can be relaxed in many ways. Why, for instance, do we assume in A2' that material varies with $r$ and not with $\alpha$ and/or $x$ ? It turns out that we can get a lot of guidance from the equations for the objective and constraint functions, as we will see below. In the process of doing so, we will also show how innovation by relaxing modeling assumptions can create the same innovations as DVE.

\subsection{A Method for Innovation by Relaxing Assumptions}

The algorithm for design innovation by relaxing modeling assumptions is presented in Figure 3. Here we explain each step of the algorithm in detail in the context of the torsion beam problem formalized in Figure 2.

Optimal solutions for constrained optimization problems in the engineering domain are often located where some inequalities (such as $g(\mathbf{x}) \leq 0$, or $g(\mathbf{x}) \geq 0$ ) are active (i.e. satisfy equality). In Step 1, one of the possible active sets is chosen. An active set consists of a subset of the inequalities (forced to satisfy equality) plus the equality constraints. The number of possible active sets is clearly exponential in the number of constraints. 
Procedure Innovate

Step 1. Apply monotonicity analysis ([Cag87]) to propose active sets and back substitute to simplify constraints and objective function.

Step 2. For each active set:

If the active set gives a feasible solution, output it.

Otherwise, do Steps 3, 4, and 5 .

Step 3. Choose a set of assumptions to relax and a form to relax each such assumption.

Step 4. Reconstruct the new set of constraints after assumption relaxation.

Step 5. Redo monotonicity analysis and back substitution, and check each resulting new active set:

- If it results in a solution, output it.

- If it is not a solution but there has been improvement, go back to Step 3 and choose an additional relaxation If no active set is either a solution or an improvement, go back to step 3 and choose a different relaxation.

Figure 3 Algorithm for Design Innovation by Assumption Relaxation

However, monotonicity analysis ([Cag87, PW79]) is used to prune this space, just as it is used in DVE. Each active set corresponds to a subspace of the space of possible designs, and the designs in different subspaces generally have different characteristics. Every design that satisfies the constraints of our problem must be in one of these subspaces.

In our torsion beam example, we apply monotonicity analysis $\$$, and find that one active set which yields physically reasonable solution is: $\{1,3, \cdots, 7\}$.

Since an active set potentially forces one or more inequality constraints to equality, we can exploit these new equations by back substitution into the constraint equations, and get more specific versions of the remaining constraints. We do back substitution and again check the monotonicity conditions, and new constraints are added to the active set if required to satisfy these conditions. After back substitution, numerical techniques (such as finite element methods) could be used if required (possibly, to approximate integrals numerically).

In the torsion beam example, for the active set above, back substitution gives the fol-

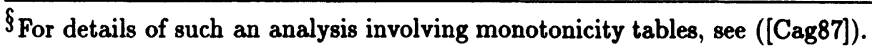


lowing expressions for the inactive constraints:

$$
W=\pi L K_{1}(G)\left(\frac{2 T}{\pi K_{2}(G)}\right)^{2 / 3} \text { and } R=\left(\frac{2 T}{\pi K_{2}(G)}\right)^{1 / 3} \text {. }
$$

For each active set produced by Step 1 , Step 2 checks if the non-active constraints are satisfied. If all non-active constraints are satisfied, we have a feasible solution which is optimally directed. "We output it, and backtrack to examine more active sets. Otherwise, we go to step 3 .

In our example, we will assume that the problem is such that there is no material (that is, no proper G), which would enable W to satisfy constraint (2. So, we go to Step 3 .

In Step 3, we propose assumptions to relax, and how to relax them. We assume the list of assumptions is known, perhaps having been recorded by the process that created the simplified models in the first place. When choosing assumptions to relax, priority is given to assumptions whose relaxation is known to improve the objective or lower the penalty associated with any unsatisfied constraint " (since we know this amounts to potential improvement of the design), followed by assumptions which affect the current active set, i.e. assumptions which mention variables in the active set. (We illustrate later on how the active set could be exploited usefully.) If several assumptions tie for the same priority explicit forms of relaxation suggested by the active set are preferred over forms used by default. Also, forms which de-simplify the model ${ }^{* *}$ minimally are preferred. For example, if the active set suggests a particular explicit form for a function that would replace a constant function because of assumption relaxation, we would prefer to use that form. Else, if the active set only suggests the variable(s) over which the function should vary, rather than an explicit form, we use a step-function over that(those) variable(s). If nothing is suggested by the active set, step-functions over different co-ordinate variables are tried in turn.

While relaxing assumptions, we relax such that we de-simplify the model as little as possible, thus trying to capture simpler designs before more complex ones. This could be achieved in two ways. First, we can identify certain relaxations as less simplifying than others. For example, while going from a constant function to the corresponding function that depends on several variables, we try functions with as few variables as possible. Thus while relaxing the homogeneity assumption we could go from constant density $\rho$ to

\footnotetext{
In optimally directed design, as defined in [CA91] page 48, one is not guaranteed of optimal solutions, but the search tries to eliminate suboptimal and dominated regions of search space while trying to improve the objective.

$\|_{\text {The model generation process could store knowledge about qualitative effects of relaxing a model as- }}$ sumption it has introduced to simplify the model.

${ }^{* *}$ we return to this criterion at the end of this section
} 
$\rho(r, \alpha, x)$. But, instead we would try $\rho$ to be function of a single variable before trying two or all three variables. Secondly, the model generation process could create a partial order among groups of assumptions based on the degree of simplification achieved. Thus the model generation process could order gases in terms of decreasing simplicity as ideal gases, Van der Waals gases and finally Redlich-Kwong gases.

To see how Step 3 applies to the example of the torsion beam, we first note that there are two assumptions: $\mathrm{A} 1$, the assumption that the beam is cylindrical with uniform radius and A2, the assumption that the beam is homogeneous. Suppose nothing is known a priori about their influence on the objective or the penalty of the unsatisfied constraint. Both assumptions, however, affect the active set, and hence, both qualify for the same priority as candidates for relaxation. Now we see if any priority arises trying to predict forms of relaxation. Relaxing A1 could yield other geometries but no particular one is suggested by the constraints in the active set. On the other hand, suppose we relax A2, the assumption of homogeneity. Recalling equation (10), and constraint (1) (from the active set) we get, $\forall r \frac{G \varphi r}{L} \leq \tau_{y}$. Under assumption A2, this was specialized to yield $\frac{G \varphi R}{L} \leq \tau_{y}$. If $\mathrm{A} 2$ is relaxed and this inequality is to be active, it immediately shows, $\tau_{y}=\frac{G \varphi r}{L}$, that is, $\tau_{y}$ is a function of $r$. (There is no guarantee that after relaxing A2 this constraint stays active, but the fact that the constraint $\forall r, \frac{G \varphi r}{L} \leq \tau_{y}(r)$ could be satisfied even when $\tau_{y}(r)<\tau(R)$ for $r<R$, could result in an improvement of the objective or some penalty.) Since choice of $\tau_{y}$ fixes the choice of the material, G and $\rho$ gets fixed too - and hence, are functions of $r$. We then have, (recalling equation (9))

$\tau_{y}=\frac{T G(r) r}{2 \pi \int G(r) r^{3} d r}$

In this case, the explicit form of the function $\tau_{y}$ after relaxation is unknown, since the explicit form of $G(r)$ is unknown. In the absence of explicit forms of functions, step functions are used to replace constant functions. DVE essentially does the same - it too proposes step functions to iteratively replace constant functions and seeks to improve the objective, gaining extra degrees of freedom from the newly introduced step parameters. Thus in this case the function for $\tau_{y}(r)$ is,

$\tau_{y}(r)= \begin{cases}\tau_{y 1} & \text { for } 0 \leq r \leq R_{1} \\ \tau_{y 2} & \text { for } R_{1} \leq r \leq R_{2}\end{cases}$

Similar step functions are proposed for $G, \rho$ etc.

In Step 4 we reconstruct the new set of constraints and relations after relaxing the assumption set suggested in Step 3. In the torsion beam example, we thus relax the assumption set $\{\mathrm{A} 2\}$. The reformulation is shown in Figure (4). 
Minimize $W=W_{1}+W_{2}$, subject to:

$$
\begin{array}{cl}
G_{1} \geq 0, & G_{2} \geq 0 \\
R_{1} \geq 0, & R_{2} \geq 0 \\
\tau_{1} \leq \tau_{y 1}, & \tau_{2} \leq \tau_{y 2} \\
W \leq W_{0} & \\
\varphi_{2} \leq \varphi_{\max } &
\end{array}
$$

where

$$
\begin{array}{cl}
W_{1}=\pi \rho_{1} L R_{1}^{2}, & W_{2}=\pi \rho_{2} L\left(R_{2}^{2}-R_{1}^{2}\right) \\
\tau_{1}=\frac{G_{1} \varphi_{1} R_{1}}{L}, & \tau_{2}=\frac{G_{2} \varphi_{2} R_{2}}{L} \\
\varphi_{1}=\varphi_{2} & \\
\varphi_{1}=\frac{2 T_{1} L}{\pi G_{1} R_{1}^{4}}, & \varphi_{2}=\frac{2 T_{2} L}{\pi G_{2}\left(R_{2}^{4}-R_{1}^{4}\right)} \\
R_{2}-R_{1}=t & \\
T_{1}+T_{2}=T & \\
\rho_{1}=K_{1}\left(G_{1}\right), & \rho_{2}=K_{1}\left(G_{2}\right) \\
\tau_{y 1}=K_{2}\left(G_{2}\right), & \tau_{y 2}=K_{2}\left(G_{2}\right)
\end{array}
$$

Figure 4 Torsional Beam Problem After Relaxation of Assumption

In step 5, we redo the monotonicity analysis, and try each of the resulting active sets to see if it is a solution to the problem, or if it at least looks like a promising step towards a solution, i.e., if either (i) the objective has improved (that is, it decreased for a minimization problem, or it increased for a maximization problem) from the previous formulation or (ii) the penalty of some inactive inequality has decreased from the previous formulation. (The penalty associated with an inequality constraint is defined as $\max (0, g(\mathbf{x}))$ for $g(\mathbf{x}) \leq 0$ and as $\operatorname{abs}(\min (0, g(\mathbf{x})))$ for $g(\mathbf{x}) \geq 0$. Essentially, it measures the 'badness' of a violated inequality constraint.)

If an active set is a solution, we output it and go on to the next active set. If an active set looks promising, we go back recursively to Step 3 and try adding a further relaxation to the current one. If none of the active sets is either a solution or even promising, backtrack to Step 3 and try a different relaxation.

In our example, after monotonicity analysis one active set that is physically relevant includes (among other constraints) $G_{1} \geq 0$ and $\tau_{2} \leq \tau_{y 2}$, but not $G_{2} \geq 0$ and $\tau_{1} \leq \tau_{y 1}$. This 
implies $\rho_{1}=0$ and $\rho_{2}>0$ (from 24), and we get a hollow tube. Using back substitution (Step 2), from (18), (24), (22) and $G_{1}=0$ we get the new objective as:

$W=\pi L K_{1}\left(G_{2}\right) t\left(2 R_{2}-t\right)$

The new objective is an improvement (over the one before relaxing the assumption) so long as:

$K_{1}\left(G_{2}\right) t\left(2 R_{2}-t\right)<K_{1}(G)\left(\frac{2 T}{\pi K_{2}(G)}\right)^{2 / 3}$

Now from $\tau_{2}=\tau_{y 2}, \tau_{y 2}=K_{2}\left(G_{2}\right), \tau_{2}=\frac{G_{2} \varphi_{2} R_{2}}{L}, \varphi_{2}=\frac{2 T_{2} L}{\pi G_{2}\left(R_{2}^{4}-R_{1}^{4}\right)}$ and $R_{2}-R_{1}=t$ we get (using back substitution):

$4 t R_{2}^{3}-6 t^{2} R_{2}^{2}+\left(4 t^{3}-\frac{2 T}{\pi K_{2}\left(G_{2}\right)}\right) R_{2}-t^{4}=0$

Since, equation (28) decreases one degree of freedom, any two of $R_{2}, G_{2}$ and $t$ can be varied to satisfy Equation (27) and hence improve the objective over the old one. The initially unsatisfied constraint (2) can now be satisfied. In a similar way, when $G_{1} \geq 0$ and $G_{2} \geq 0$ are both inactive, it can be shown that the step functions proposed devise a composite rod with an improved objective.

\section{ADVANTAGES OF ASSUMPTION RELAXATION}

The previous section shows how a typical innovation derived by DVE can also be derived by relaxing modeling assumptions.

DVE can be viewed as a special case of the general principle of assumption relaxation. As a result of relaxing assumptions one introduces more design variables into the problem formulation (in particular, for DVE, these are the step-parameters), thereby extending the search space. In this section we illustrate how the general principle improves over DVE in at least two ways.

\subsection{Direct Suggestion of Solutions in the Limit}

We consider the role of the active set of constraints in suggesting relaxations. We show that by considering these, we can arrive at the same designs which DVE achieves as the limit of several of its iterations by inducing a trend over those iterations. Our approach is more direct, and since it avoids the DVE iterations whenever possible, achieves the same 
Minimize W, subject to:

$$
\begin{gathered}
\sigma \leq \sigma_{y} \\
\tau \leq \tau_{y} \\
W \leq W_{0} \\
G \geq 0 \\
R \geq 0
\end{gathered}
$$

where

$$
\begin{gathered}
\tau=\frac{P}{\pi R^{2}} \\
\sigma=\frac{4 P x}{\pi R^{3}} \\
W=\pi \rho L R^{2} \\
\rho=K_{1}(G) \\
\tau_{y}=K_{2}(G) \\
\sigma_{y}=K_{3}(G)
\end{gathered}
$$

and

$P$ is the transverse load

$\sigma$ is the bending stress at distance $\mathbf{x}$

$\sigma_{y}$ is the bending stress at yield

and, the other parameters correspond to those in Figure 1

Figure 5 The Problem of Beam Under Bending Load

results faster. We illustrate this by considering a variation (Figure 5 ) of the example considered earlier. A beam, of length $L$ having minimum weight is to be designed, such that it is clamped at one end and subject to a transverse load $P$ at the other end.

Assumptions (A1) and (A2) both carry over from the previous example. Consider the case where bending stress is dominant. This corresponds to the active set $\{29,34, \cdots$, 39 \}, and leads to

$$
R=\left(\frac{4 P L}{\pi K_{3}(G)}\right)^{1 / 3}
$$


$W=\pi L K_{1}(G)\left(\frac{4 P L}{\pi K_{3}(G)}\right)^{2 / 3}$

Suppose, given $P$ and $L$, no choice of material (that is, no proper G), enables W to satisfy constraint (31). We seek hints from the active set to see if some assumptions could be relaxed usefully.

The active set implies

$\sigma_{y}=\frac{4 P x}{\pi R^{3}}$

Based on this we could relax either of the assumptions. Relaxing only (A1) (but not A2), leads to $R$ as a function of $x$ :

$R=\left(\frac{4 P x}{\pi \sigma_{y}}\right)^{1 / 3}$

so long as, constraint (29) remains active (that is bending is dominant). But supposing that constraint (30) is active instead of constraint (29) (that is, shear stress is dominant, rather than bending stress), the radius is given by

$R=\left(\frac{P}{\pi \tau_{y}}\right)^{1 / 2}$

One should note at this point that it is quite possible for different active sets to dictate solutions over different regions over the artifact (just like it is possible to have the same active set dictate the solution over the whole artifact.) One should simply explore all such possibilities to generate solutions, and finally keep only the ones physically relevant. The interesting solution in this example has different active sets dominating different regions over the co-ordinate variable $x$. The point of crossover $^{\dagger \dagger}$ is found by equating these two expressions for $\mathrm{R}$, giving $x_{c}=\frac{\pi \sigma_{y}}{4 P}\left(\frac{P}{\pi \tau_{y}}\right)^{3 / 2}$. Thus, for $x \leq x_{c}$ (that is, for regions nearer to free end) shear stress dominates and the radius is given by equation (44); whereas, for $x \geq x_{c}$ (that is, for regions nearer to the clamped end) bending stress dominates and equation (43) gives the corresponding $R$. Since the new $R$ is a strict underestimate of the previous $\mathrm{R}$ given by equation (40), the new $\mathrm{W}$ will also be lower than before.

It should be noted that this is exactly the solution proposed after several iterations of DVE followed by an 'inductive leap'. The design proposed by such an inductive leap needs to be validated, since it is not guaranteed to be correct. Our method proposes exactly the same solution, and just like DVE, needs to be validated. But in proposing the solution

$\dagger_{\text {where the active set changes. }}$ 
we avoid the several iterations of DVE. This is significant because of the way in which active sets are chosen. Given $m$ constraints there are $2^{m}$ ways of choosing active sets. Monotonicity analysis prunes this space, and reduces the choice to a lower number. But higher the number of equations, higher the number of choices. In each iteration of DVE, $(n-1) u_{p}$ additional constraints are added, where $\mathrm{n}$ is the number of regions to which a single region expands and $u_{p}$ is the number of parallel constraints in a region before the expansion. So after several iterations of DVE, the process becomes computationally expensive. As acknowledged in ([CA91]), some constraints do not dominate for several iterations, and induction by DVE based on too few iterations would wrongly predict those constraints to be inactive. That is why in DVE, even though certain constraints are deemed inductively active, all constraints need to be considered across the continuum of induced trend to check for constraint violations. Our heuristic of relaxing assumptions based on suggestions from the active set can reduce several iterations of DVE, and hence is computationally more attractive.

What if we relaxed assumption (A2) instead of assumption (A1) ? Going back to equation (42) (under bending dominance) we find that relaxation of assumption A2 suggests $\sigma_{y}$ to be a function of $x$ according to the right hand side $\sharp$ of that equation. For the region before the crossover point, that is, for $x \leq x_{c}$, shear stress is dominant, and hence the density $\rho_{1}$ is determined $\S^{\S}$ by $\tau_{y}=\frac{P}{\pi R^{2}}$. For the region beyond the crossover point, bending stress dominates and $\rho$ gets chosen (via $\sigma_{y}$ ) according to equation (42). Following equation (8) the objective is now given by

$W=\pi R^{2} x_{c} \rho_{1}+\pi R^{2} \int_{x_{c}}^{L} \rho(x) d x$

For the region where bending stress is active, $\sigma_{y}(x)$ is a strict underestimate over its old counterpart $\sigma_{y}$, and since $\sigma_{y}$ and $\rho$ are monotonically related because of equations (37) and (39), it follows that the new $\rho(x)$ would underestimate the old $\rho$. The contribution to the objective by the region $x \geq x_{c}$ (as given by the second term on the right hand side of equation 45) would be strictly less than earlier, and hence there is a good chance that the objective decreases as well.

\footnotetext{
$\$ \ddagger$ numerical techniques could be employed to evaluate the integral involved since $\rho(x)$ might not be known in closed form or could be too complicated

$\S \S$ There is only 1 degree of freedom between density $\rho$, yield shear stress $\tau_{y}$ and yield bending stress $\sigma_{y}$, since choice of any one effectively fixes choice of the material.
} 


\subsection{Importing Implicit Variables}

In this section we show how our approach is more general compared to DVE. We present an example where relaxing model assumptions leads to introduction of variables in the search space which is not possible by DVE. DVE essentially duplicates variables (dimensional, system and region variables) over the regions it expands to. What if no explicit variables remain (to get duplicated) because of model simplification? DVE can't regenerate them. The intuition is that because of model simplification some design variables may have gotten lost which are not related to dimensional variables (or more fundamentally, coordinate variables) in any way in the de-simplified model we get after relaxation, and DVE can't handle such cases.

Consider the following design problem. You are given a spring (of spring modulus $k>0$ ) attached to a fixed support at one end, with a mass $m$ at the other end. The mass moves along a single dimension, under an external force $F$ on it. $F=k_{1}+k_{2} \sin (\omega t)$, where $\mathrm{t}$ is time and $k_{1}, k_{2}$ are constants greater than 0 . The objective is to minimize mass $m$ subject to the constraints: (i) the mass $m$ is below a threshold, (ii)after a long time, the amplitude $\alpha$ does not grow beyond a given threshold $\alpha_{0}$, and (iii) $\omega$ is not lower than the natural frequency $\omega_{N}$ by more than a very small fixed threshold $\delta$.

The problem is formally stated below. Minimize the mass $\mathrm{m}$ subject to the constraints $\mathcal{C}:$

mass : $m \leq m_{0}$

amplitude : $\alpha \leq \alpha_{0}$

frequency : $0<\omega_{N}-\omega \leq \delta$

The displacement at time $\mathrm{t}$ is the superposition of $x_{g}$ and $x_{p}$ \% given by:

$x_{g}=-\frac{k_{1} \cos \left(\omega_{N} t\right)}{k}-\frac{\omega k_{2} \sin \left(\omega_{N} t\right)}{\omega_{N} k\left(1-\frac{\omega^{2}}{\omega_{N}^{2}}\right)}$

and

$x_{p}=\frac{k_{2} \sin (\omega t)}{k\left(1-\frac{\omega^{2}}{\omega_{N}^{2}}\right)}+\frac{k_{1}}{k}$

Tी these are respectively the general and particular solutions of the governing differential equation: $m \frac{d^{2} x}{d t^{2}}+k x=F$ 
where,

naturalfrequency $: \omega_{N}=\sqrt{\frac{k}{m}}$

When $\omega_{N}-\omega \leq \delta$ is active, it follows from equations (49) and (50) that since $\delta$ is a small quantity, the magnitude of both $x_{g}$ and $x_{p}$, and hence the amplitude, is large. In fact the fixed quantity $\delta$ could be so small that the amplitude is sufficiently large to violate constraint (47).

We seek to lower the penalty associated with the violated constraint (47). We relax the original assumption of no damping since the model generation process could note that such a relaxation would reduce the amplitude (and hence the penalty associated with the violated constraint (47)). Note that, in this case, the active constraint does not by itself involve any assumptions. In case the model generation process did not produce the annotation of the effect of relaxing this assumption, we would anyway relax the assumption and see the consequence in the next iteration.

We thus relax this assumption of no damping, and add to the model:

Damping force : $F_{D}=-k_{D} \frac{d x}{d t}$

where $k_{D}>0$ is the damping parameter.

The displacement for large $t$, is now :

$x_{p}=\frac{k_{1}}{k}+A \cos (\omega t-\phi)$

where,

$A=\frac{k_{2}}{\sqrt{k^{2}\left(1-\left(\frac{\omega}{\omega_{N}}\right)^{2}\right)^{2}+\left(k_{D} \omega\right)^{2}}}$

and,

$\tan (\phi)=-\left(\frac{k-m \omega^{2}}{k_{D} \omega}\right)$

Assuming $\omega_{N}-\omega \leq \delta$ is active, even though we still have a difference of only $\delta$ between $\omega_{N}$ and $\omega$, the amplitude $A$ in equation (54) can be reduced by suitably choosing $k_{D}$. Thus it follows that the newly introduced variable $k_{D}$ lets us reduce the penalty associated with the violated constraint (47) to zero and allows a feasible design.

We have shown earlier that DVE is essentially the result of relaxing certain types of 
simplifying assumptions about the model. Expansion of a dimensional variable is tantamount to allowing variation of properties over one or more co-ordinate variable which was not allowed in the earlier model. But what about non-dimensional variables lost due to simplifying assumptions? For example, in the case of the oscillator, the simplifying assumption of no damping results in no trace of $k_{D}$ in the model; by relaxing that assumption we can introduce it and expand our search space to generate new designs, not possible in the earlier model. This is clearly not possible in DVE.

We should note here, that our design task consists of assigning values to design parameters. How they are actually realized is another issue. Thus we can think of a different mechanism (maybe human assisted) which knows how to actually compose the design given these design parameters. Thus given radius, length and the material the 'composer' knows how to put together the cylinder. When a damping parameter is introduced, the 'composer' is expected to know how to achieve this damping (maybe introducing friction etc.). The actual working of such an operation is beyond the scope of the current work.

\section{RELATION TO OTHER WORK}

Aside from the work on Dimensional Variable Expansion, there are several other pieces of work that are related to the ideas reported here.

PROMPT ([MA87]) stores large amount of physics knowledge in its Graph of Models structure (described in more detail in ([ACP91]). Each node represents a model of the system being analyzed and the edges represent the differing approximations between two nodes. Search switches between nodes in the graph when prediction of a model fails to meet observations. PROMPT uses the interesting idea of a case library of precompiled heuristics, known as modification operators, to modify a prototype. Example of such an operator would be one for redistributing mass from areas of low stress to areas of high stress, plus some applicability conditions for such an operator. While use of precompiled human experience imparts better design capabilities and leads to efficiency, by the same token, modifications not specified by heuristics cannot take place. Functional relationships between parameters are not utilized. Furthermore, the system does not reason with inequality constraints to optimize the design. Our method resembles this work in essentially creating various models based on differences of modeling assumptions and searching in different models; but, there are differences with our approach. For one, the representation for models differ in granularity - our's is more fine grained. We do not use human compiled heuristics, and hence our method does not suffer from the resulting drawback mentioned above. We do utilize functional relationships between parameters and we also 
reason with inequality constraints to push the design towards optimality. Given the task of beam design under torsional load, DVE designs the composite beam, but PROMPT cannot achieve it. Our method achieves the same design as DVE does in this task.

[FF91] describe a method for model composition. They compose a model sufficient to answer a query while minimizing extraneous detail from a general domain theory and a structural description of a specific system. They decompose a domain theory into a set of model fragments and deduce a set of assumptions from the requirements of the query. Then they use an ATMS to find a minimum set of model fragments sufficient to satisfy the query. This work ([FF91]) is closely related to [ACP91] and it relates to our work in that we too try to find the simplest model that allows us to design the artifact. Their task at hand is, however, different from ours: they take the structural description of a system and compose a model to satisfy the query; we, seek to design the structure such that it behaves in a particular way. Our approach also differs in the way solutions are searched. We search in a simplified model first, and if we hit an impasse in that model, we relax some assumptions and go to a more complex model. Their approach is to synthesize a minimal but sufficient model.

[Wel92] presents a framework for dynamically changing model accuracy based on model sensitivity analysis. Their goal is to mechanize the process of crafting underlying models of complex physical systems by building a model management system that evaluates simplifying assumptions and selects appropriate perspectives. Our work is a step in the same direction in the sense that it shows when and how to relax simplifying assumptions opportunistically to come up with innovative designs.

[LSJ92] and [LS90] describe a system called MSG, which generates mathematical models to analyze physical systems involved in heat transfer behavior. It uses strong domain theory to guide model construction. It first identifies regions of interest on an object, then determines relevant heat transfer and energy storage processes and finally transforms these processes into equations. A set of choices for the models in terms of control volumes, and heat flows are to be made and a related paper ([LS93]) describes reduction operators used to make these choices. Various rough models are used to estimate the physical parameters on which these rules depend. The connection of this work with our work is in the fact that both recognize the need for modeling underlying physics at various levels of approximation. While MSG uses model simplification to guide model generation, our approach exploits the assumptions recorded by such a process to guide design innovation. Both approaches underscore the importance of models of varying levels of simplicity. 


\section{CONCLUSION}

We have proposed a method for innovation in design based on relaxing modeling assumptions. Engineering design problems are generally formalized as constrained optimization problems where the objective function and the constraints refer to several properties of the resulting design. While setting up such a problem, several assumptions about the model are made to narrowly define the problem context. Our key observation is that such a process could import assumptions that constrain the problem too much to find a feasible solution. By relaxing appropriate modeling assumptions we can modify the search space profitably. We have shown that the method of DVE, found in the literature, is a special case of this. We have also shown that in some of the cases where DVE proposes a solution by induction, our method proposes the same solution with less computational effort. We have also shown that our method generalizes DVE to include design innovations based on reimporting (non-dimensional) variables lost originally due to model simplification. We observe that the model generation process could enhance the power of this method by introducing assumptions incrementally and storing information as to how the assumptions influence the design.

\section{ACKNOWLEDGEMENTS}

The work presented here is part of the 'Hypercomputing \& Design' (HPCD) project, and is supported by ARPA under contract DABT-63-93-C-0064. The content of the information herein does not necessarily reflect the position of the Government and official endorsement should not be inferred.

Thanks are due to Prof. Haim Baruh for his useful suggestions on the mass and spring problem.

\section{REFERENCES}

[ACP91] S. Addanki, R. Cremonini, and J.S. Penberthy. Graphs of models. Artificial Intelligence, 51:145-177, 1991.

[ACP92] V. Aelion, J. Cagan, and G. Powers. Input variable expansion - an algorithmic design generation technique. Research in Engineering Design, 4:101-113, 1992.

[CA91] J. Cagan and A. M. Agogino. Inducing constraint activity in innovative design. AI EDAM, 5(1):47-61, 1991.

[Cag87] J. Cagan. Innovative design of mechanical structures from first principles. $A I$ 
EDAM, 1(3):169-189, 1987.

[Cag91] J. Cagan. Dimensional variable expansion - a formal approach to innovative design. Research in Engineering Design, 3:75-85, 1991.

[FF91] Brian Falkenhainer and Kenneth D. Forbus. Compositional modeling: Finding the right model for the job. Artificial Intelligence, 51:95-143, 1991.

[LS90] S. R. Ling and L. Steinberg. Automated generation of approximate models for thermal casting. In Working Notes of the AAAI-90 workshop on Automatic Generation of Approximations and Abstraction, 1990.

[LS93] S. R. Ling and L. Steinberg. Approximation operators in distributed modeling. Technical Report cap-tr-15, Dept. of Computer Science, Rutgers University, New Brunswick, NJ 08903, U.S.A., 1993.

[LSJ92] S. R. Ling, L. Steinberg, and Y. Jaluria. Msg: A computer system for automated modeling of heat transfer. Technical Report cap-tr-18, Dept. of Computer Science, Rutgers University, New Brunswick, NJ 08903, U.S.A., 1992.

[MA87] S. S. Murthy and S. Addanki. Prompt : An innovative design tool. In Proceedings of AAAI-87, pages 637-642, Seattle, WA, July 13-17 1987.

[MZG89] M. L. Maher, F. Zhao, and J. S Gero. An approach to knowledge-based creative design. In Preprints of NSF Engineering Design Research Conference, pages 333-346. College of Engineering, Univ. Of Massachussets, Amherst, 1989.

[PW79] P. Papalambros and D.J. Wilde. Global non-iterative design optimization using monotonicity analysis. Journal of Mechanical Design, 101:645-649, 1979.

[Wel92] D. S. Weld. Reasoning about model accuracy. Artificial Intelligence, 56, 1992.

\section{BIOGRAPHY}

Jyotibikas Bhattacharya is in the Ph.D. program in the Department of Computer Science at Rutgers University. He has a Master of Science degree from the same department and a Master of Technology degree from I.I.T., Kanpur, India. Currently, he is looking into various aspects of engineering design, with emphasis on design innovation. His research interests include Artificial Intelligence, Software Engineering and Database Systems.

Louis I. Steinberg is an Associate Professor of Computer Science at Rutgers University New Brunswick, and Associate Director of the High Performance Computing and Design (HPCD) project. His areas of research interest include knowledge-based approaches to design and design aids, machine learning, other areas of artificial intelligence, and digital halftoning. He earned a BS degree in computer science in 1972 from the University of 
Illinois at Urbana/Champaign and a $\mathrm{PhD}$ in computer science from Stanford University in 1980 . 